\title{
EQUILIBRATED STRESS SPACE FOR NONLINEAR DIMENSIONALLY REDUCED SHELL MODELS IN TERMS OF FIRST-ORDER STRESS FUNCTIONS
}

\author{
EDGÁR BERTÓtI \\ Institute of Applied Mechanics, University of Miskolc \\ H-3515 Miskolc-Egyetemváros, Hungary \\ edgar . bertoti@uni-miskolc.hu
}

[Received: August 18, 2020; Accepted: September 26, 2020]

Dedicated to Professor Barna Szabó on the occasion of his eighty-fifth birthday

\begin{abstract}
Considering the power series expansion of the three-dimensional variables with respect to the shell thickness coordinate, an equilibrated stress space for the first PiolaKirchhoff stress vectors is derived in convective curvilinear coordinate system. The infinite series of the two-dimensional translational equilibrium equations are satisfied by introducing two first-order stress function vectors expanded into power series. For the important case of thin shells, the infinite number of two-dimensional equilibrium equations is truncated to obtain a 'first-order' model, where the equilibrated stress-space requires three vectorial stress function coefficients only. The formulation presented for thin shells is compared to the nonlinear equilibrium equations of the classical shell theories, written in terms of the first Piola-Kirchhoff stress resultants and stress couples and satisfied by the introduction of three first-order stress function vectors.
\end{abstract}

Mathematical Subject Classification: 05C38, 15A15

Keywords: shell theory, equilibrated stress space, first-order stress functions

\section{INTRODUCTION}

A self-equilibrated stress space in elasticity can be generated by stress functions through differentiation. A divergence-free second-order stress tensor requires firstorder stress functions and the stress components are obtained by the combinations of their first-order derivatives. When the symmetry of the stress tensor is also a priori required, second-order stress functions are needed. In that case the stress components are generated by the combinations of the second-order derivatives of the stress functions.

The terminology 'first-order' and 'second-order' for describing stress functions has been introduced by Fraeijs de Veubeke [1-3]. A self-equilibrated divergence-free stress space requires six non-zero components of the tensor of first-order stress functions 2 , 4. 5], whereas a symmetric and divergence-free stress space requires three non-zero components of a second-order stress-function tensor $[2,6]$. The main advantage in the use of first-order stress functions, from the point of view of finite element analysis, is that they require $C_{0}$-continuous approximations, in contrast to the $C_{1}$-continuity 
requirement for second-order stress functions. In addition, the use of first-order stress functions "not only simplifies the construction of stress elements by a reduction to $C_{0}$ continuity but opens the field to the three- or two-dimensional stress elements with curved boundaries" [2].

The stress function approach and the related complementary energy-based variational principles have been successfully applied in the finite element analysis of twodimensional elasticity problems, see, e.g., [2, 7, 9]. For three-dimensional problems, however, no efficient finite element model exists, which is due mainly to the $C_{1}$ continuity requirement for the second-order stress functions on the one hand, and to the structure of the zero-energy modes and the difficulties with their suppression for the case of first-order stress functions, on the other hand [10].

Complementary energy-based equilibrium models using stress functions have also been developed for structural members like plates and shells. Considering the analogy between the special forms of the linear kinematic equations and the equilibrium equations for shells, stress functions were introduced for cylindrical shells by Schaefer [11] and for general shells by Günther [12 and Goldenveizer 13]. The relationship between those shell stress functions and the Maxwell-Morera-type second-order stress functions has been investigated by Yamamoto 14]. Based on the Kirchhoff-Love kinematical assumption, a nonlinear shell theory with finite rotation and stress function vectors was proposed by Simmonds and Danielson [15] and later, using alternate stress and conjugate strain measures, by Atluri [16]. In those works the membrane equilibrium equation is satisfied by one first-order stress function vector and the bending strains are described by one rotation vector. The mixed variational principles proposed in those works rely on both the bending strain energy and the complementary membrane energy of the shell.

An equilibrium finite element model for plates has been developed by Punch and Atluri [17. The intrinsic shell theory formulated by Valid [18 is also based on the Kirchhoff-Love kinematical assumption and assumes the existence of a two-dimensional surface complementary strain energy density. The linearized equilibrium equations, written in the terms of stress resultants and stress couples, are satisfied by the introduction of a first-order and a second-order stress function vector. For the nonlinear equilibrium equations of the shell theory considered in [18], however, "every attempt to find SA (statically admissible) stresses would be illusory", as stated on page 75 of [18. Dual-mixed $h p$ finite element models for cylindrical shells using first-order stress functions and rotations have been developed in [19] and 20.

The main goal of this paper is to derive an equilibrated stress space for nonlinear shell problems without any a priori-made kinematical assumptions for the deformation. Considering the shell as a three-dimensional body, the starting point of the derivation is the three-dimensional equilibrium equations for the first Piola-Kirchhoff stress vectors. The dimensional reduction procedure is based on the Taylor-series expansions of the variables, describing the geometry and the mechanical state of the shell, with respect to the thickness coordinate.

In Section 2 the basic concepts and the notation necessary for understanding the paper, are summarized. Section 3 presents a dimensional reduction procedure for the 
derivation of an equilibrated stress space for the first Piola-Kirchhoff stress vectors in convective curvilinear frame, attached to the shell middle surface. The infinite series of the two-dimensional translational equilibrium equations, written in terms of the expanded stress coefficients, are satisfied by introducing two first-order stress function vectors expanded into power series. The equilibrated stress spaces are given for two cases: equilibrium without and with satisfied stress boundary conditions on the shell faces. Section 4 considers the important case of thin shells, for which a 'firstorder model' is derived. The equilibrated stress space for the finite number of stress coefficients is compared to the equilibrated stress space derived for stress resultants and stress couples of the classical shell models, and the relationships between the first-order stress function vectors are derived.

\section{Geometric PRELiminaries}

This section summarizes some fundamental concepts used in shell theories and introduces the notation applied throughout the paper. Both invariant and index notations are applied. When the index notation is used, the summation convention is applied. Latin indices, as usual, take the range of values 1,2,3 and the Greek indices of the values 1 and 2 . The scalar product between two tensors is indicated by one dot. The tensorial or dyadic product between two tensors of any order has no special sign.

2.1. Reference domain. To describe the deformation of the shell as a three-dimensional (3D) body, we define first a simply connected two-dimensional (2D) reference domain, a plain denoted by $\bar{S}$, which is bounded by a sufficiently smooth boundary curve $\bar{\ell}$ and parametrized by the coordinates $\xi^{\alpha}$. Let a 3D reference domain be defined as

$$
V=\left\{\boldsymbol{\xi}\left|\xi^{\alpha} \in \bar{S},\right| \xi^{3} \mid<d / 2\right\},
$$

where the coordinate $\xi^{3}$ is measured along a straight line perpendicular to the coordinate lines $\xi^{\alpha}$ at each point of $\bar{S}$, and $d$ is the thickness of the domain (Figure 1 ). It is assumed that $d$ is independent of $\xi^{\alpha}$, i.e., the thickness of $V$ is constant. The surface $\bar{S}$ described by the equation $\xi^{3}=0$ is called the middle surface of $V$. The boundary surface of $V$, denoted by $S$, consists of the top and bottom surfaces

$$
S^{ \pm}=\left\{\boldsymbol{\xi} \mid \xi^{\alpha} \in \bar{S}, \xi^{3}= \pm d / 2\right\}
$$

and the lateral surface

$$
S^{\times}=\left\{\boldsymbol{\xi}\left|\xi^{\alpha} \in \bar{\ell},\right| \xi^{3} \mid \leq d / 2\right\}
$$

for which the relations $S=S^{ \pm} \cup S^{\times}, S^{ \pm} \cap S^{\times}=\emptyset$ hold.

In the subsequent analysis it is assumed that an arbitrary tensor quantity $\boldsymbol{T}\left(\xi^{i}\right)$, defined at the point $\xi^{i} \in V$, can be differentiated sufficient number of times with respect to $\xi^{3}$ and can be expanded into Taylor-series around the point $\xi^{3}=0$ :

$$
\begin{aligned}
\boldsymbol{T}\left(\xi^{i}\right) & =\boldsymbol{T}\left(\xi^{\alpha}, 0\right)+\left.\frac{\partial \boldsymbol{T}}{\partial \xi^{3}}\right|_{\xi^{3}=0} \xi^{3}+\left.\frac{1}{2} \frac{\partial^{2} \boldsymbol{T}}{\partial\left(\xi^{3}\right)^{2}}\right|_{\xi^{3}=0}\left(\xi^{3}\right)^{2}+\cdots \\
& ={ }_{0} \boldsymbol{T}\left(\xi^{\alpha}\right)+{ }_{1} \boldsymbol{T}\left(\xi^{\alpha}\right) \xi^{3}+{ }_{2} \boldsymbol{T}\left(\xi^{\alpha}\right)\left(\xi^{3}\right)^{2}+\cdots=\sum_{n=0}^{\infty}{ }_{n} \boldsymbol{T}\left(\xi^{\alpha}\right)\left(\xi^{3}\right)^{n}
\end{aligned}
$$


where the left subscript $n=0,1,2, \ldots$ refers to the coefficient of the $n$-th power of $\xi^{3}$. Note that the coefficients ${ }_{n} \boldsymbol{T}\left(\xi^{\alpha}\right), n=0,1,2, \ldots$ depend on the coordinates $\xi^{\alpha}$ only.

2.2. Initial and current configuration of the shell. The initial and the current configuration of the shell are denoted by ${ }^{0} \mathrm{~V}$ and ${ }^{t} V$, respectively, the material points of which are labeled by the Cartesian coordinates $X^{A}$ and $x^{a}$ (Figure 1). The 3D domains ${ }^{0} V$ and ${ }^{t} V$ are obtained as one-to-one nonlinear mappings from the reference configuration $V$, according to the equations

$$
\boldsymbol{X}=\boldsymbol{X}(\boldsymbol{\xi}), \quad X^{A}=X^{A}\left(\xi^{i}\right)
$$

and

$$
\boldsymbol{x}=\boldsymbol{x}(\boldsymbol{\xi}, t), \quad x^{a}=x^{a}\left(\xi^{i}, t\right),
$$

where $\xi^{i}$ are the convective coordinates. The deformation of the shell is described by the nonlinear mapping

$$
\boldsymbol{x}=\boldsymbol{x}(\boldsymbol{X}, t), \quad x^{a}=x^{a}\left(X^{A}, t\right) .
$$

The relationships between the mapping functions (2.5)- 2.7) are given by $\boldsymbol{x}(\boldsymbol{\xi}, t)=$ $\boldsymbol{x}[\boldsymbol{X}(\boldsymbol{\xi}), t]$ and $\boldsymbol{x}(\boldsymbol{X}, t)=\boldsymbol{x}\left[\boldsymbol{\xi}^{-1}(\boldsymbol{X}), t\right]$. The faces of the shell in the initial and the

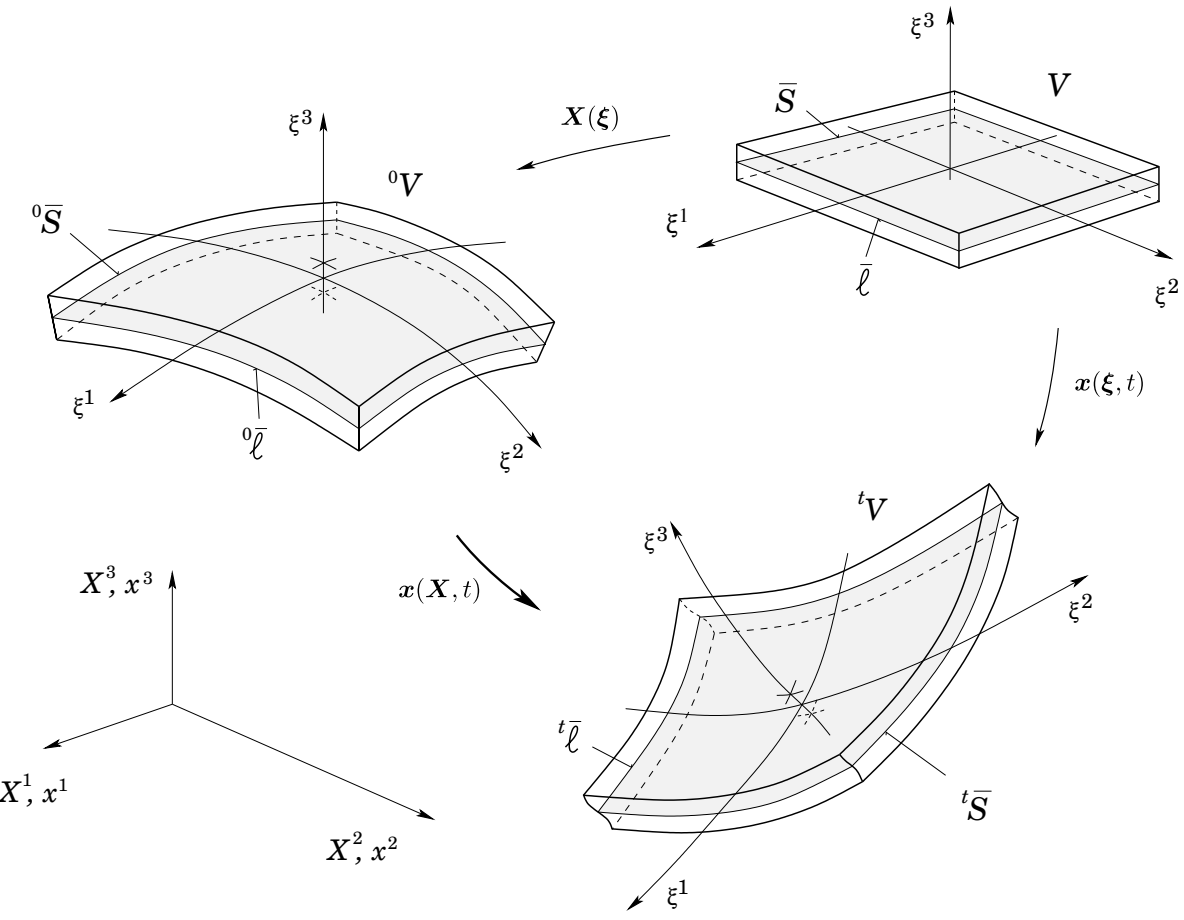

Figure 1. Initial and current configurations of the shell

current configuration are denoted, respectively, by ${ }^{0} S^{ \pm}$and ${ }^{t} S^{ \pm}$, the lateral surfaces by ${ }^{0} S^{\times}$and ${ }^{t} S^{\times}$, the middle surfaces by ${ }^{0} \bar{S}$ and ${ }^{t} \bar{S}$, and their boundary curves by ${ }^{0} \bar{\ell}$ 
and ${ }^{t} \bar{\ell}$ (Figure 1). The unit normals to the shell faces are denoted by ${ }^{0} \boldsymbol{n}^{ \pm}$and ${ }^{t} \boldsymbol{n}^{ \pm}$, and those to the lateral surfaces by ${ }^{0} \boldsymbol{n}^{\times}$and ${ }^{t} \boldsymbol{n}^{\times}$.

Applying the material description of the deformation, the mapping 2.5 that defines the initial configuration ${ }^{0} V$ is assumed, without loss of generality, to be a linear function of $\xi^{3}$ :

$$
\boldsymbol{X}\left(\xi^{i}\right)={ }_{0} \boldsymbol{X}\left(\xi^{\alpha}\right)+{ }_{1} \boldsymbol{X}\left(\xi^{\alpha}\right) \xi^{3} .
$$

The coordinate lines $\xi^{3}$ in ${ }^{0} V$ are straight and assumed to be perpendicular to the middle surface ${ }^{0} \bar{S}$. The mapping $(2.6)$ that gives the current configuration ${ }^{t} V$ is, in general, a non-linear function of $\xi^{3}$ and can be given as

$$
\boldsymbol{x}\left(\xi^{i}\right)={ }_{0} \boldsymbol{x}\left(\xi^{\alpha}\right)+{ }_{1} \boldsymbol{x}\left(\xi^{\alpha}\right) \xi^{3}+{ }_{2} \boldsymbol{x}\left(\xi^{\alpha}\right)\left(\xi^{3}\right)^{2}+\ldots
$$

with an unknown number of coefficients ${ }_{n} \boldsymbol{x}\left(\xi^{\alpha}\right), n=0,1,2, \ldots$. This means that the coordinate line $\xi^{3}$ in ${ }^{t} V$ is usually neither straight nor perpendicular to the deformed middle surface ${ }^{t} \bar{S}$.

Remark. Classical plate and shell models are usually based on kinematical assumptions that can be considered as restrictions to the mapping $(2.6)$ and its Taylor series (2.9). The Reissner-Mindlin plate model [21, 22] and the Naghdi shell model [23, 24], for example, assume that the mapping $(2.9)$ is linear in $\xi^{3}$. The Kirchhoff-plate model 25 and the Love-Novozhilov-Sanders-Koiter shell model 26 29 assume, in addition, that the coordinate line $\xi^{3}$ is perpendicular to the deformed middle surface, according to the Kirchhoff-Love hypothesis.

2.3. Convective base vectors. The convective covariant base vectors in the initial configuration are given by

$$
\boldsymbol{A}_{a}\left(\xi^{i}\right)=\frac{\partial \boldsymbol{X}}{\partial \xi^{a}}
$$

which, using (2.8), can be written as

$$
\begin{aligned}
& \boldsymbol{A}_{\alpha}=\frac{\partial_{0} \boldsymbol{X}}{\partial \xi^{\alpha}}+\frac{\partial_{1} \boldsymbol{X}}{\partial \xi^{\alpha}} \xi^{3}={ }_{0} \boldsymbol{A}_{\alpha}\left(\xi^{\alpha}\right)+{ }_{1} \boldsymbol{A}_{\alpha}\left(\xi^{\alpha}\right) \xi^{3}, \\
& \boldsymbol{A}_{3}= \\
& { }_{1} \boldsymbol{X}\left(\xi^{\alpha}\right)={ }_{0} \boldsymbol{A}_{3}\left(\xi^{\alpha}\right),
\end{aligned}
$$

where ${ }_{0} \boldsymbol{A}_{a}\left(\xi^{\alpha}\right)$ denotes the (convective, covariant) tangent base vectors to the middle surface ${ }^{0} \bar{S}$ (see Figure 2). According to 2.11)-2.12, the variation of $\boldsymbol{A}_{\alpha}$ in $\xi^{3}$ is linear, whereas $\boldsymbol{A}_{3}={ }_{0} \boldsymbol{A}_{3}$ is constant in $\xi^{3}$. Since the coordinate line $\xi^{3}$ is perpendicular to the middle surface ${ }^{0} \bar{S}, \boldsymbol{A}_{3}={ }_{0} \boldsymbol{A}_{3}$ is the unit normal to the surfaces $\xi^{3}=$ constant in the initial configuration.

Taking into account 2.12 , the coefficients ${ }_{1} \boldsymbol{A}_{\alpha}$ in the power series $(2.11)$ can be expressed by the base vectors ${ }_{0} \boldsymbol{A}_{\beta}$ as

$$
{ }_{1} \boldsymbol{A}_{\alpha}=\frac{\partial_{1} \boldsymbol{X}}{\partial \xi^{\alpha}}=\frac{\partial_{0} \boldsymbol{A}_{3}}{\partial \xi^{\alpha}}=-B_{\alpha{ }_{0}}^{\beta} \boldsymbol{A}_{\beta}
$$

where

$$
\boldsymbol{B}=B_{\alpha}^{\beta}{ }_{0} \boldsymbol{A}_{\beta{ }_{0}} \boldsymbol{A}^{\alpha}=-{ }_{1} \boldsymbol{A}_{\alpha{ }_{0}} \boldsymbol{A}^{\alpha}, \quad B_{\alpha}^{\beta}=-{ }_{1} \boldsymbol{A}_{\alpha} \cdot{ }_{0} \boldsymbol{A}^{\beta}
$$




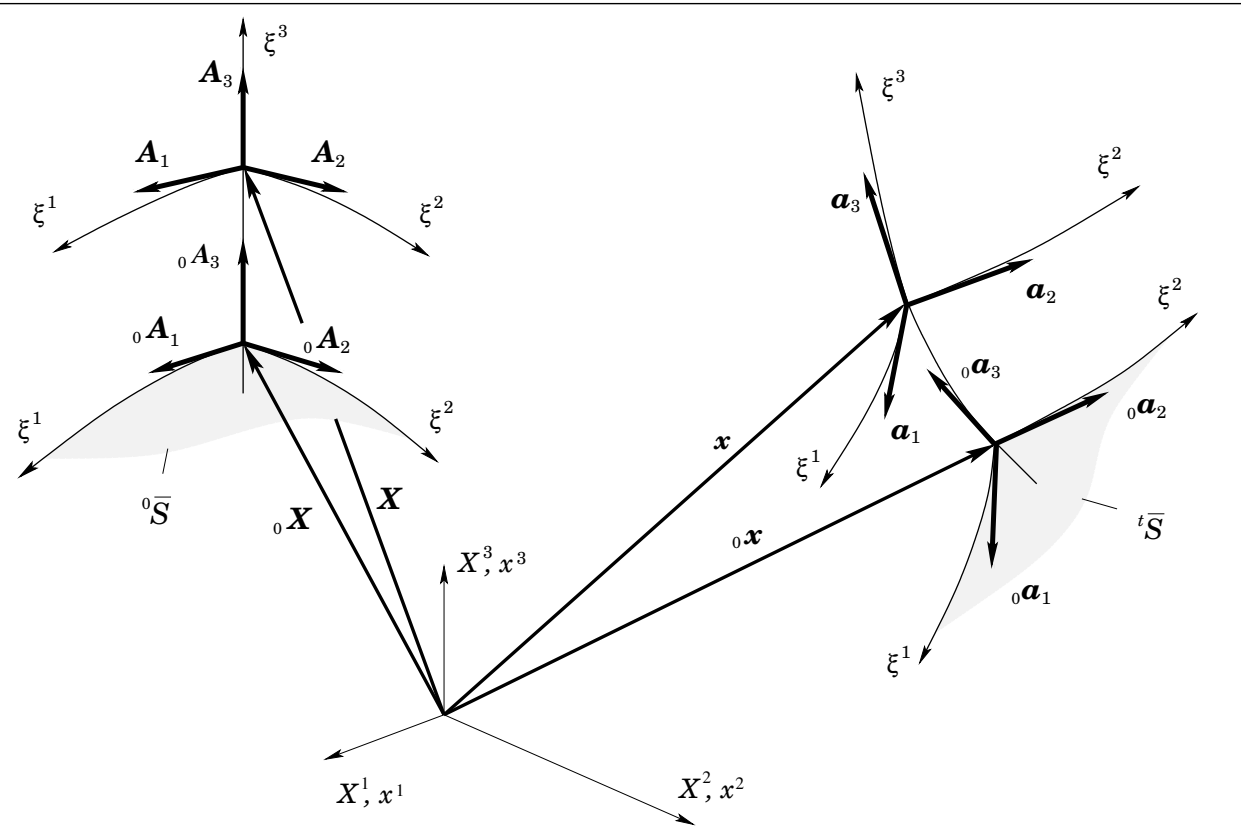

Figure 2. Base vectors in the reference and the current configuration

is the (symmetric) curvature tensor of the middle surface ${ }^{0} \bar{S}$. Taking into account 2.13 , equation 2.11) can be rewritten in the form

$$
\boldsymbol{A}_{\alpha}=\left(\delta_{\alpha}^{\beta}-B_{\alpha}^{\beta} \xi^{3}\right){ }_{0} \boldsymbol{A}_{\beta} .
$$

Introducing the shifter tensor as

$$
\begin{gathered}
\mu_{a}^{b}=\boldsymbol{A}_{a} \cdot{ }_{0} \boldsymbol{A}^{b}, \quad \mu_{\alpha}^{\beta}=\delta_{\alpha}^{\beta}-B_{\alpha}^{\beta} \xi^{3}, \quad \mu_{3}^{\beta}=\mu_{\alpha}^{3}=0, \quad \mu_{3}^{3}=1, \\
\boldsymbol{\mu}=\mu_{a}^{b}{ }_{0}{ }_{0} \boldsymbol{A}_{b{ }_{0}} \boldsymbol{A}^{a}=\boldsymbol{A}_{a{ }_{0}} \boldsymbol{A}^{a}
\end{gathered}
$$

and its inverse

$$
\left(\mu^{-1}\right)_{a}^{b}={ }_{0} \boldsymbol{A}_{a} \cdot \boldsymbol{A}^{b}, \quad \boldsymbol{\mu}^{-1}=\left(\mu^{-1}\right)_{a}^{b} \boldsymbol{A}_{b} \boldsymbol{A}^{a}={ }_{0} \boldsymbol{A}_{a} \boldsymbol{A}^{a} \quad \boldsymbol{\mu} \cdot \boldsymbol{\mu}^{-1}=\mathbf{1},
$$

the relationships between the covariant base vectors 2.12 and 2.15 can be given by

$$
\boldsymbol{A}_{a}=\mu_{a}^{b}{ }_{0} \boldsymbol{A}_{b}, \quad{ }_{0} \boldsymbol{A}_{a}=\left(\mu^{-1}\right)_{a}^{b} \boldsymbol{A}_{b},
$$

and those between the contravariant base vectors by

$$
\boldsymbol{A}^{b}=\left(\mu^{-1}\right)_{a{ }_{0}}^{b} \boldsymbol{A}^{a}, \quad{ }_{0} \boldsymbol{A}^{b}=\mu_{a}^{b} \boldsymbol{A}^{a} .
$$

The determinant of the shifter $\boldsymbol{\mu}$ in terms of the curvature tensor is given by

$$
\mu=\operatorname{det} \boldsymbol{\mu}=1-(\operatorname{tr} \boldsymbol{B}) \xi^{3}+(\operatorname{det} \boldsymbol{B})\left(\xi^{3}\right)^{2},
$$

where $\operatorname{tr} \boldsymbol{B}=B_{\alpha}^{\alpha}$ and $\operatorname{det} \boldsymbol{B}$ is the determinant of $\boldsymbol{B}$. For thin shells the approximations

$$
\boldsymbol{\mu}=\mathbf{1}, \quad \mu_{a}^{b}=\delta_{a}^{b}, \quad \mu=1
$$


can be applied for the shifter. The derivative of the shifter 2.16 and its determinant 2.21 with respect to $\xi^{3}$ are proportional to the curvature of the shell, i.e., they cannot be neglected either for thin shells.

The covariant components of the metric tensor at the arbitrary point of the shell in the initial configuration are given by $A_{k l}=\boldsymbol{A}_{k} \cdot \boldsymbol{A}_{l}$, and on the middle surface ${ }^{0} \bar{S}$ by ${ }_{0} A_{k l}={ }_{0} \boldsymbol{A}_{k} \cdot{ }_{0} \boldsymbol{A}_{l}$. Their determinants, denoted by $A=\operatorname{det} A_{k l}$ and ${ }_{0} A=\operatorname{det}{ }_{0} A_{k l}$, respectively, are related to each other by

$$
\sqrt{A}=\mu \sqrt{{ }_{0} A},
$$

where $\sqrt{A}$ is also known as the Jacobian of the mapping $\boldsymbol{X}=\boldsymbol{X}(\boldsymbol{\xi})$. Using the contravariant metric components $A^{i j}$ and ${ }_{0} A^{i j}$, the relationships between the coand contravariant base vectors are given by

$$
\boldsymbol{A}^{i}=A^{i j} \boldsymbol{A}_{j}, \quad{ }_{0} \boldsymbol{A}^{i}={ }_{0} A^{i j}{ }_{0} \boldsymbol{A}_{j} .
$$

The convective covariant base vectors in the current configuration are defined by

$$
\boldsymbol{a}_{p}\left(\xi^{i}\right)=\frac{\partial \boldsymbol{x}}{\partial \xi^{p}} .
$$

Taking into account $(2.9)$, they can be written as

$$
\boldsymbol{a}_{p}\left(\xi^{i}\right)={ }_{0} \boldsymbol{a}_{p}\left(\xi^{\alpha}\right)+{ }_{1} \boldsymbol{a}_{p}\left(\xi^{\alpha}\right) \xi^{3}+{ }_{2} \boldsymbol{a}_{p}\left(\xi^{\alpha}\right)\left(\xi^{3}\right)^{2}+\ldots,
$$

where ${ }_{0} \boldsymbol{a}_{p}\left(\xi^{\alpha}\right)$ are the base vectors on the deformed middle surface ${ }^{t} \bar{S}$ (Figure 2). As can be seen from 2.26), $\boldsymbol{a}_{p}$ is, in general, a nonlinear function of $\xi^{3}$. When the deformation gradient

$$
\boldsymbol{F}=\frac{\partial \boldsymbol{x}}{\partial \boldsymbol{X}}=\boldsymbol{a}_{p} \boldsymbol{A}^{p}
$$

is known, the convective base vectors in the current configuration can be obtained as

$$
\boldsymbol{a}_{p}=\boldsymbol{F} \cdot \boldsymbol{A}_{p}, \quad \boldsymbol{a}^{q}=\boldsymbol{F}^{-T} \cdot \boldsymbol{A}^{q} .
$$

\section{Equilibrated StRESS SPACE OF THE SHELL}

Nonlinear dual-mixed finite element models based on equilibrated stress spaces can be constructed by satisfying the translational equilibrium equation for the first Piola-Kirchhoff stress tensor through the introduction of first-order stress functions. The rotational equilibrium, i.e., the symmetry of the Cauchy stress tensor, is satisfied in a weak sense through the variational formulation and by the corresponding finite element model. For shells with arbitrary geometry, the construction of an equilibrated stress space in convective curvilinear coordinates requires special considerations. As will be shown in this section, the key issue is the use of the vectorial form of the translational equilibrium equation in the shell coordinate space. The Taylor-series expansion of the stress vectors leads to a sequence of two-dimensional translational equilibrium equations that can be satisfied by introducing first-order stress function vectors. 


\subsection{Vectorial equilibrium equations.}

3.1.1. Translational equilibrium. The three-dimensional translational equilibrium equation for the first Piola-Kirchhoff stress tensor $\boldsymbol{P}=P^{k \ell} \boldsymbol{a}_{k} \boldsymbol{A}_{\ell}$ in the convective coordinate system is given by

$$
\boldsymbol{P} \cdot{ }^{0} \nabla+{ }^{0} \rho \boldsymbol{b}=\mathbf{0} \quad \boldsymbol{X} \in{ }^{0} V,
$$

where

$$
{ }^{0} \nabla=\boldsymbol{A}^{i} \frac{\partial}{\partial \xi^{i}}
$$

is the differential operator in the initial configuration, ${ }^{0} \rho$ is the mass density in the undeformed state and $\boldsymbol{b}$ is the prescribed body force density per unit mass. Introducing the first Piola-Kirchhoff stress vectors as

$$
\boldsymbol{p}^{\ell}=\boldsymbol{P} \cdot \boldsymbol{A}^{\ell}, \quad \boldsymbol{P}=\boldsymbol{p}^{\ell} \boldsymbol{A}_{\ell}
$$

and making use of the identity

$$
\boldsymbol{P} \cdot{ }^{0} \nabla=\frac{\partial \boldsymbol{p}^{\ell}}{\partial \xi^{\ell}}+\frac{1}{\sqrt{A}} \frac{\partial \sqrt{A}}{\partial \xi^{\ell}} \boldsymbol{p}^{\ell}
$$

for the divergence of the second-order tensor $\boldsymbol{P}$ (see, e.g., [30]), the equilibrium equation $(3.1)$ can be rewritten in vectorial form:

$$
\frac{1}{\sqrt{A}}\left(\sqrt{A} \boldsymbol{p}^{\ell}\right)_{, \ell}+{ }^{0} \rho \boldsymbol{b}=\mathbf{0} \quad \boldsymbol{X} \in{ }^{0} V
$$

Note that equation (3.1) contains the covariant derivative of the stress components $P^{k \ell}$, whereas the vectorial equation (3.5) contains the partial derivative of the stress vectors $\boldsymbol{p}^{\ell}$. This is an important fact when the construction of an equilibrated stress space is needed in a general curvilinear coordinate frame.

3.1.2. Rotational equilibrium. The symmetry of the Cauchy stress tensor $\boldsymbol{\sigma}=J^{-1} \boldsymbol{P}$. $\boldsymbol{F}^{T}$ in terms of the first Piola-Kirchhoff stress vectors is expressed by the vectorial equation

$$
\boldsymbol{a}_{\ell} \times \boldsymbol{p}^{\ell}=\mathbf{0},
$$

where $\boldsymbol{a}_{\ell}$ are the base vectors defined by 2.25 . Satisfaction of the rotational equilibrium equation (3.6) is not required a priori in the stress-based dual-mixed shell model under consideration, it is given here only for the sake of completeness.

3.2. Equilibrated stress vectors. To derive an equilibrated space for the first Piola-Kirchhoff stress vectors $\boldsymbol{p}^{\ell}$ in the convective coordinate system, the threedimensional translational equilibrium equation $\sqrt{3.5}$ is rewritten first by making use of 2.23$)$ :

$$
\frac{1}{\sqrt{{ }_{0} A}}\left(\sqrt{{ }_{0} A} \mu \boldsymbol{p}^{\ell}\right), \ell+{ }^{0} \rho \mu \boldsymbol{b}=\mathbf{0}
$$

in which $\sqrt{{ }_{0} A}$ depends on $\xi^{\alpha}$ only. The stress vectors $\mu \boldsymbol{p}^{\ell}\left(\xi^{i}\right)$ and the body force vector ${ }^{0} \rho \mu \boldsymbol{b}$ are expanded next into power series with respect to the coordinate $\xi^{3} \equiv \zeta$ :

$$
\hat{\boldsymbol{p}}^{\ell}:=\mu \boldsymbol{p}^{\ell}\left(\xi^{\alpha}, \zeta\right)=\sum_{n=0}^{\infty}{ }_{n} \hat{\boldsymbol{p}}^{\ell}\left(\xi^{\alpha}\right) \zeta^{n},
$$




$$
\hat{\boldsymbol{b}}:={ }^{0} \rho \mu \boldsymbol{b}\left(\xi^{\alpha}, \zeta\right)=\sum_{n=0}^{\infty}{ }_{n} \hat{\boldsymbol{b}}\left(\xi^{\alpha}\right) \zeta^{n} .
$$

Inserting (3.8) and (3.9) in (3.7), the three-dimensional equilibrium equation is replaced by a series of two-dimensional equations, according to the powers of $\zeta$ :

$$
\frac{1}{\sqrt{{ }_{0} A}}\left({\sqrt{{ }_{0}} A}_{n} \hat{\boldsymbol{p}}^{\lambda}\right)_{, \lambda}+(n+1)_{n+1} \hat{\boldsymbol{p}}^{3}+{ }_{n} \hat{\boldsymbol{b}}=\mathbf{0}, \quad n=0,1,2,3, \ldots
$$

Equilibrium equations in 3.10 can be satisfied by introducing two arbitrary but differentiable first-order stress function vectors, denoted by $\boldsymbol{\psi}$ and $\chi$, and expanding them into power series with respect to $\zeta$ as

$$
\begin{aligned}
& \psi\left(\xi^{\alpha}, \zeta\right)=\sum_{n=0}^{\infty}{ }_{n} \psi\left(\xi^{\alpha}\right) \zeta^{n}, \\
& \chi\left(\xi^{\alpha}, \zeta\right)=\sum_{n=0}^{\infty}{ }_{n} \chi\left(\xi^{\alpha}\right) \zeta^{n} .
\end{aligned}
$$

Then the equilibrium equations 3.10 are identically satisfied by the following stress vectors:

$$
\begin{aligned}
& { }_{n} \hat{\boldsymbol{p}}^{\lambda}={ }_{0} \epsilon^{\lambda \beta}{ }_{n} \boldsymbol{\psi}_{, \beta}-(n+1){ }_{0} \epsilon^{3 \lambda}{ }_{n+1} \boldsymbol{\chi}-\frac{1}{2 \sqrt{{ }_{0} A}}{ }_{n} \boldsymbol{f}^{\lambda}, \quad n=0,1,2,3, \ldots, \\
& { }_{n} \hat{\boldsymbol{p}}^{3}={ }_{0} \epsilon^{3 \beta}{ }_{n} \boldsymbol{\chi} \boldsymbol{}_{, \beta}, \quad n=1,2,3,4, \ldots,
\end{aligned}
$$

where ${ }_{0} \epsilon^{\lambda \beta}$ and ${ }_{0} \epsilon^{3 \beta}$ are two-dimensional permutation symbols defined by

$$
\sqrt{{ }_{0} A}{ }_{0} \epsilon^{\lambda \beta}=\left\{\begin{array}{r}
1, \text { if } \lambda=1, \beta=2 \\
-1, \text { if } \lambda=2, \beta=1
\end{array}, \quad \sqrt{0}_{0}{ }_{0} \epsilon^{3 \beta}=\left\{\begin{array}{r}
1, \text { if } \beta=1 \\
-1, \text { if } \beta=2
\end{array},\right.\right.
$$

and

$$
{ }_{n} \boldsymbol{f}^{\lambda}=\int_{\left(\xi^{\lambda}\right)} \sqrt{0}_{0} \hat{\boldsymbol{b}} \mathrm{d} \xi^{\lambda}, \quad n=0,1,2, \ldots
$$

are known load vectors obtained from the prescribed body forces by integration along the coordinate lines $\xi^{1}$ and $\xi^{2}$. Note that ${ }_{0} \hat{\boldsymbol{p}}^{3}$ does not appear in 3.10 and, therefore, in 3.14, its value is arbitrary, at least from the point of view of equilibrium.

Dual-mixed finite element models based on the above stress space require the satisfaction of the stress boundary conditions on the top and bottom faces, as well as on the lateral surfaces of the shell. These boundary conditions can be satisfied by applying the Lagrange multiplier technique, i.e., using hybridized elements.

\subsection{Equilibrated stress vectors with satisfied stress boundary conditions on}

the faces. In the classical, dimensionally reduced shell models it is generally assumed that on the top and bottom faces of the shells only surface loads are present and the prescribed tractions are naturally included in the equilibrium equations written in terms of stress resultants and stress couples. Considering the equilibrium equations in the form (3.10), the prescribed loads on the faces of the shell can be taken into account as follows. 
3.3.1. Stress boundary conditions on the faces of the shell. The stress boundary conditions for the first Piola-Kirchhoff stress vectors (3.3) at the top and bottom faces ${ }^{0} S^{ \pm}$of the shell with outward unit normals ${ }^{0} \boldsymbol{n}^{ \pm}= \pm \boldsymbol{A}^{3}= \pm \boldsymbol{A}_{3}$ are given by

$$
\pm \boldsymbol{p}^{3}={ }^{0} \widetilde{\boldsymbol{p}}^{ \pm} \quad \boldsymbol{X} \in{ }^{0} S^{ \pm},
$$

and on the lateral surface ${ }^{0} S_{p}^{\times}$with outward unit normal ${ }^{0} \boldsymbol{n}^{\times}={ }^{0} n_{\lambda}^{\times} \boldsymbol{A}^{\lambda}$ is given by

$$
{ }^{0} n_{\lambda}^{\times} \boldsymbol{p}^{\lambda}={ }^{0} \widetilde{\boldsymbol{p}}^{\times} \quad \boldsymbol{X} \in{ }^{0} S_{p}^{\times},
$$

where ${ }^{0} \widetilde{\boldsymbol{p}}^{ \pm}$and ${ }^{0} \widetilde{\boldsymbol{p}}^{\times}$are prescribed tractions.

3.3.2. Vectorial equilibrium including the surface load components. In view of the left side of (3.8), the stress boundary conditions (3.17), prescribed on the top and bottom faces of the shell for the first Piola-Kirchhoff stress vector $\boldsymbol{p}^{3}$, take the form

$$
\pm \hat{\boldsymbol{p}}^{3}=\mu^{ \pm 0} \widetilde{\boldsymbol{p}}^{ \pm} \quad \boldsymbol{X} \in{ }^{0} S^{ \pm}
$$

which, taking into account 3.8 , is rewritten as

$$
{ }_{0} \hat{\boldsymbol{p}}^{3}+\frac{d}{2}{ }_{1} \hat{\boldsymbol{p}}^{3}+\left(\frac{d}{2}\right)^{2}{ }_{2} \hat{\boldsymbol{p}}^{3}+\ldots+\left(\frac{d}{2}\right)^{n}{ }_{n} \hat{\boldsymbol{p}}^{3}+\ldots= \pm \mu^{ \pm 0} \widetilde{\boldsymbol{p}}^{ \pm} .
$$

Subtracting and adding the two equations corresponding to the signs \pm in $(3.20)$, the equations

$$
\begin{aligned}
& { }_{0} \hat{\boldsymbol{p}}^{3}+\left(\frac{d}{2}\right)^{2}{ }_{2} \hat{\boldsymbol{p}}^{3}+\left(\frac{d}{2}\right)^{4}{ }_{4} \hat{\boldsymbol{p}}^{3}+\ldots=\frac{1}{2}\left(\mu^{+0} \widetilde{\boldsymbol{p}}^{+}-\mu^{-0} \widetilde{\boldsymbol{p}}^{-}\right), \\
& { }_{1} \hat{\boldsymbol{p}}^{3}+\left(\frac{d}{2}\right)^{2}{ }_{3} \hat{\boldsymbol{p}}^{3}+\left(\frac{d}{2}\right)^{4}{ }_{5} \hat{\boldsymbol{p}}^{3}+\ldots=\frac{1}{d}\left(\mu^{+0} \widetilde{\boldsymbol{p}}^{+}+\mu^{-0} \widetilde{\boldsymbol{p}}^{-}\right)
\end{aligned}
$$

are obtained. For the sake of compact notation, let the load vector components

$$
\begin{aligned}
{ }_{0} \widetilde{\boldsymbol{p}}\left(\xi^{\alpha}\right) & =\frac{1}{2}\left(\mu^{+0}{ }^{0} \widetilde{\boldsymbol{p}}^{+}-\mu^{-0}{ }^{0} \widetilde{\boldsymbol{p}}^{-}\right), \\
{ }_{1} \widetilde{\boldsymbol{p}}\left(\xi^{\alpha}\right) & =\frac{1}{d}\left(\mu^{+0} \widetilde{\boldsymbol{p}}^{+}+\mu^{-0} \widetilde{\boldsymbol{p}}^{-}\right)
\end{aligned}
$$

be introduced. Then the load vector defined by

$$
\widetilde{\boldsymbol{p}}={ }_{0} \widetilde{\boldsymbol{p}}+{ }_{1} \widetilde{\boldsymbol{p}} \zeta
$$

at $\zeta= \pm d / 2$ takes the values of the prescribed loads on the top and bottom surfaces:

$$
\widetilde{\boldsymbol{p}}\left(\xi^{\alpha}, \pm d / 2\right)= \pm \mu^{ \pm 0} \widetilde{\boldsymbol{p}}^{ \pm} .
$$

Taking into account 3.23)-3.24, the coefficients ${ }_{1} \hat{\boldsymbol{p}}^{3}$ and ${ }_{2} \hat{\boldsymbol{p}}^{3}$ can be expressed from the stress boundary conditions (3.21)-(3.22) as

$$
\begin{aligned}
{ }_{1} \hat{\boldsymbol{p}}^{3} & ={ }_{1} \widetilde{\boldsymbol{p}}-\sum_{i=1}^{\infty}\left(\frac{d}{2}\right)^{2 i}{ }_{2 i+1} \hat{\boldsymbol{p}}^{3}, \\
{ }_{2} \hat{\boldsymbol{p}}^{3} & =\frac{4}{d^{2}}\left({ }_{0} \widetilde{\boldsymbol{p}}-{ }_{0} \hat{\boldsymbol{p}}^{3}\right)-\sum_{i=1}^{\infty}\left(\frac{d}{2}\right)^{2 i}{ }_{2 i+2} \hat{\boldsymbol{p}}^{3} .
\end{aligned}
$$


Inserting them in the first two equilibrium equations of 3.10 that correspond to $n=0$ and $n=1$, the translational equilibrium equations including the prescribed loads on the faces are given by

$$
\begin{array}{ll}
n=0: & \frac{1}{\sqrt{{ }_{0} A}}\left(\sqrt{{ }_{0} A}{ }_{0} \hat{\boldsymbol{p}}^{\lambda}\right)_{, \lambda}-\sum_{i=1}^{\infty}\left(\frac{d}{2}\right)^{2 i}{ }_{2 i+1} \hat{\boldsymbol{p}}^{3}+{ }_{1} \widetilde{\boldsymbol{p}}+{ }_{0} \hat{\boldsymbol{b}}=\mathbf{0}, \\
n=1: & \frac{1}{\sqrt{{ }_{0} A}}\left(\sqrt{{ }_{0} A}{ }_{1} \hat{\boldsymbol{p}}^{\lambda}\right)_{, \lambda}-2 \sum_{i=1}^{\infty}\left(\frac{d}{2}\right)^{2 i}{ }_{2 i+2} \hat{\boldsymbol{p}}^{3}+\frac{8}{d^{2}}\left({ }_{0} \widetilde{\boldsymbol{p}}-{ }_{0} \hat{\boldsymbol{p}}^{3}\right)+{ }_{1} \hat{\boldsymbol{b}}=\mathbf{0}, \\
n \geq 2: & \frac{1}{\sqrt{{ }_{0} A}}\left(\sqrt{{ }_{0} A}{ }_{n} \hat{\boldsymbol{p}}^{\lambda}\right)_{, \lambda}+(n+1)_{n+1} \hat{\boldsymbol{p}}^{3}+{ }_{n} \hat{\boldsymbol{b}}=\mathbf{0} .
\end{array}
$$

Equations (3.31) for the case $n \geq 2$ are identical with those in 3.10 . Note also that the value of ${ }_{0} \hat{\boldsymbol{p}}^{3}$ is not arbitrary in this case: due to $(3.20)$ and (3.28), it appears in the equilibrium equations.

3.3.3. Equilibrated stress vectors. Equilibrium equations $(3.29)-(3.31)$ can identically be satisfied by the stress function vectors $\psi$ and $\chi$, introduced in Section 3.2 . Considering their power series expansion $3.11-3.12$ with respect to $\xi^{3} \equiv \zeta$, the equilibrated stress vectors are given by

$$
\begin{aligned}
& { }_{0} \hat{\boldsymbol{p}}^{\lambda}={ }_{0} \epsilon^{\lambda \beta}{ }_{0} \boldsymbol{\psi}_{, \beta}-{ }_{0} \epsilon^{3 \lambda} \sum_{i=1}^{\infty}\left(\frac{d}{2}\right)^{2 i}{ }_{2 i+1} \boldsymbol{\chi}-\frac{1}{2 \sqrt{{ }_{0} A}}{ }_{1} \boldsymbol{f}^{\lambda}, \\
& { }_{1} \hat{\boldsymbol{p}}^{\lambda}={ }_{0} \epsilon^{\lambda \beta}{ }_{1} \boldsymbol{\psi}_{, \beta}+\frac{8}{d^{2}}{ }_{0} \epsilon^{3 \lambda}{ }_{0} \boldsymbol{\chi}-2{ }_{0} \epsilon^{3 \lambda} \sum_{i=1}^{\infty}\left(\frac{d}{2}\right)^{2 i}{ }_{2 i+2} \chi-\frac{1}{2 \sqrt{{ }_{0} A}}{ }_{0} \boldsymbol{f}^{\lambda}, \\
& { }_{n} \hat{\boldsymbol{p}}^{\lambda}={ }_{0} \epsilon^{\lambda \beta}{ }_{n} \boldsymbol{\psi}_{, \beta}-(n+1){ }_{0} \epsilon^{3 \lambda}{ }_{n+1} \boldsymbol{\chi}-\frac{1}{2 \sqrt{{ }_{0} A}}{ }_{n} \boldsymbol{f}^{\lambda}, \quad n=2,3,4, \ldots, \\
& { }_{n} \hat{\boldsymbol{p}}^{3}={ }_{0} \epsilon^{3 \lambda}{ }_{n} \boldsymbol{\chi}_{, \lambda}, \quad n=0,3,4,5, \ldots,
\end{aligned}
$$

where

$$
\begin{array}{r}
{ }_{0} \boldsymbol{f}^{\lambda}=\int_{\left(\xi^{\lambda}\right)} \sqrt{{ }_{0} A}\left(\frac{8}{d^{2}}{ }_{0} \widetilde{\boldsymbol{p}}+{ }_{1} \hat{\boldsymbol{b}}\right) \mathrm{d} \xi^{\lambda}, \\
{ }_{1} \boldsymbol{f}^{\lambda}=\int_{\left(\xi^{\lambda}\right)} \sqrt{{ }_{0} A}\left({ }_{1} \tilde{\boldsymbol{p}}+{ }_{0} \hat{\boldsymbol{b}}\right) \mathrm{d} \xi^{\lambda}
\end{array}
$$

are known load vectors obtained from the prescribed body forces and surface loads by integration along the coordinate lines $\xi^{1}$ and $\xi^{2}$. The load vectors ${ }_{n} \boldsymbol{f}^{\lambda}, n=2,3, \ldots$. in (3.34) are defined by (3.16) and the permutation symbols ${ }_{0} \epsilon^{\lambda \beta}$ and ${ }_{0} \epsilon^{3 \beta}$ by (3.15).

Note that since the stress vectors ${ }_{1} \hat{\boldsymbol{p}}^{3}$ and ${ }_{2} \hat{\boldsymbol{p}}^{3}$ are computed in this case from the stress boundary conditions on the faces, according to (3.27) and (3.28), the equilibrated stress space $3.32-3.35$ does not require the use of the stress function coefficients ${ }_{1} \chi$ and ${ }_{2} \chi$. This means that the finite element models based on the stress space (3.32)-(3.35), with satisfied stress boundary condition on the faces, are more efficient than those based on (3.13) and (3.14) of Section 3.2. The fact that the number of the unknown stress function components is less by 6 for the present case has 
a special advantage in the modeling of thin shells, where a first-order approximation with two equilibrium equations from (3.10) is usually enough for an accurate solution (see Section 4).

3.4. Equilibrated stress components. The equilibrated components of the first Piola-Kirchhoff stress tensor can be obtained from the definitions of the stress vectors (3.3) and (3.8). Introducing the components of the stress vector $\hat{\boldsymbol{p}}^{\ell}$ with respect to the base vectors ${ }_{0} \boldsymbol{A}_{k}$ as

$$
\hat{\boldsymbol{p}}^{\ell}=\hat{P}^{k \ell}{ }_{0} \boldsymbol{A}_{k}, \quad \hat{P}^{k \ell}=\hat{\boldsymbol{p}}^{\ell} \cdot{ }_{0} \boldsymbol{A}^{k}=\mu \boldsymbol{p}^{\ell} \cdot{ }_{0} \boldsymbol{A}^{k},
$$

the power series of $\hat{\boldsymbol{p}}^{\ell}$ in 3.8 can be rewritten as

$$
\hat{\boldsymbol{p}}^{\ell}=\sum_{n=0}^{\infty}{ }_{n} \hat{\boldsymbol{p}}^{\ell} \zeta^{n}=\sum_{n=0}^{\infty}{ }_{n} \hat{P}^{k \ell}{ }_{0} \boldsymbol{A}_{k} \zeta^{n},
$$

from which the coefficients ${ }_{n} \hat{\boldsymbol{p}}^{\ell}$ are obtained as

$$
{ }_{n} \hat{\boldsymbol{p}}^{\ell}={ }_{n} \hat{P}^{k \ell}{ }_{0} \boldsymbol{A}_{k}, \quad n=0,1,2,3, \ldots .
$$

Considering the stress function vectors (3.11) and 3.12 in the expanded forms

$$
\begin{aligned}
& \boldsymbol{\psi}=\psi^{k}{ }_{0} \boldsymbol{A}_{k}=\left(\sum_{n=0}^{\infty}{ }_{n} \psi^{k} \zeta^{n}\right){ }_{0} \boldsymbol{A}_{k}, \\
& \boldsymbol{\chi}=\chi^{k}{ }_{0} \boldsymbol{A}_{k}=\left(\sum_{n=0}^{\infty}{ }_{n} \chi^{k} \zeta^{n}\right){ }_{0} \boldsymbol{A}_{k},
\end{aligned}
$$

the equilibrated stress coefficients ${ }_{n} \hat{P}^{k \ell}$ for the case of Section 3.2 are obtained by inserting (3.40)-(3.42) in (3.13) and (3.14):

$$
\begin{aligned}
& { }_{n} \hat{P}^{k \lambda}={ }_{0} \epsilon^{\lambda \beta}{ }_{n} \psi_{\mid \beta}^{k}-(n+1){ }_{0} \epsilon^{3 \lambda}{ }_{n+1} \chi^{k}-\frac{1}{2 \sqrt{{ }_{0} A}}{ }_{n}\left(f^{k}\right)^{\lambda}, \quad n=0,1,2,3, \ldots, \\
& { }_{n} \hat{P}^{k 3}={ }_{0} \epsilon^{3 \beta}{ }_{n} \chi^{k}{ }_{\mid \beta}, \quad n=1,2,3,4, \ldots,
\end{aligned}
$$

where ${ }_{n}\left(f^{k}\right)^{\lambda}, n=0,1,2,3, \ldots$ are the components of the load vectors $(3.16)$ with respect to the base vectors ${ }_{0} \boldsymbol{A}_{k}$, and the sign $\mid$ in the right subscript followed by $\beta$ refers to the three-dimensional covariant derivative on the middle surface ${ }^{0} \bar{S}$ with respect to $\xi^{\beta}$ :

$$
{ }_{n} \psi_{\mid \beta}^{k}={ }_{n} \psi_{, \beta}^{k}+{ }_{n} \psi^{\ell} \bar{\Gamma}_{\ell \beta}^{k}
$$

with Christoffel-symbols $\bar{\Gamma}_{\ell m}^{k}={ }_{0} \boldsymbol{A}_{\ell, m}{ }_{0} \boldsymbol{A}^{k}$, defined on the middle surface of the shell in the initial configuration.

The components of the equilibrated stress space for the case of Section 3.3 can be obtained from equations $3.32-(3.35)$ by inserting $(3.40)-3.42$ in them:

$$
\begin{aligned}
& { }_{0} \hat{P}^{k \lambda}={ }_{0} \epsilon^{\lambda \beta}{ }_{0} \psi^{k \beta}{ }_{\mid \beta}-{ }_{0} \epsilon^{3 \lambda} \sum_{i=1}^{\infty}\left(\frac{d}{2}\right)^{2 i}{ }_{2 i+1} \chi^{k}-\frac{1}{2 \sqrt{{ }_{0} A}{ }_{1}\left(f^{k}\right)^{\lambda},} \\
& { }_{1} \hat{P}^{k \lambda}={ }_{0} \epsilon^{\lambda \beta}{ }_{1} \psi_{\mid \beta}^{k}+\frac{8}{d^{2}}{ }_{0} \epsilon^{3 \lambda}{ }_{0} \chi^{k}-2{ }_{0} \epsilon^{3 \lambda} \sum_{i=1}^{\infty}\left(\frac{d}{2}\right)^{2 i}{ }_{2 i+2} \chi^{k}-\frac{1}{2 \sqrt{{ }_{0} A}}{ }_{0}\left(f^{k}\right)^{\lambda},
\end{aligned}
$$




$$
\begin{aligned}
& { }_{n} \hat{P}^{k \lambda}={ }_{0} \epsilon^{\lambda \beta}{ }_{n} \psi_{\mid \beta}^{k}-(n+1){ }_{0} \epsilon^{3 \lambda}{ }_{n+1} \chi^{k}-\frac{1}{2 \sqrt{{ }_{0} A}}{ }_{n}\left(f^{k}\right)^{\lambda}, \quad n=2,3,4, \ldots, \\
& { }_{n} \hat{P}^{k 3}={ }_{0} \epsilon^{3 \lambda}{ }_{n} \chi^{k}{ }_{\mid \lambda}, \quad n=0,3,4,5, \ldots,
\end{aligned}
$$

where ${ }_{n}\left(f^{k}\right)^{\lambda}, n=0,1,2,3, \ldots$ are the components of the load vectors 3.36-3.37 and $(3.16)$ with respect to the base vectors ${ }_{0} \boldsymbol{A}_{k}$. The stress components ${ }_{1} P^{k 3}$ and ${ }_{2} \hat{P}^{k 3}$ are obtained from equations $3.27-3.28$ :

$$
\begin{aligned}
{ }_{1} \hat{P}^{k 3} & ={ }_{1} \tilde{p}^{k}-\sum_{i=1}^{\infty}\left(\frac{d}{2}\right)^{2 i}{ }_{2 i+1} \hat{P}^{k 3}, \\
{ }_{2} \hat{P}^{k 3} & =\frac{4}{d^{2}}\left({ }_{0} \tilde{p}^{k}-{ }_{0} \hat{P}^{k 3}\right)-\sum_{i=1}^{\infty}\left(\frac{d}{2}\right)^{2 i}{ }_{2 i+2} \hat{P}^{k 3} .
\end{aligned}
$$

The shifted components $\bar{P}^{k m}$ of the first Piola-Kirchhoff stress tensor with respect to the base vectors ${ }_{0} \boldsymbol{A}_{i}$ can be obtained using the definition

$$
\boldsymbol{P}=\bar{P}^{k m}{ }_{0} \boldsymbol{A}_{k 0}{ }_{0} \boldsymbol{A}_{m}
$$

and the relations $3.3,3.8,3.38$ and 2.19 :

$$
\boldsymbol{P}=\boldsymbol{p}^{\ell} \boldsymbol{A}_{\ell}=\frac{1}{\mu} \hat{\boldsymbol{p}}^{\ell} \boldsymbol{A}_{\ell}=\frac{1}{\mu} \hat{P}^{k \ell}{ }_{0} \boldsymbol{A}_{k} \boldsymbol{A}_{\ell}=\frac{1}{\mu} \mu_{\ell}^{m} \hat{P}^{k \ell}{ }_{0} \boldsymbol{A}_{k 0} \boldsymbol{A}_{m} .
$$

On comparing $(3.52)$ and 3.53 the relation

$$
\bar{P}^{k m}=\frac{1}{\mu} \mu_{\ell}^{m} \hat{P}^{k \ell}
$$

is obtained. The expanded coefficients of the equilibrated stress components $\bar{P}^{k \ell}$ can be derived from (3.54) by inserting (3.43)-3.44) or 3.46- 3.51). The resulting, rather lengthy, expressions are not listed here.

\section{The SPECIAL CASE OF THIN SHELLS}

In the previous sections there was no limitation on the thickness of the shell. The number of the stress coefficients in the power series has been considered to be infinite, which resulted in an infinite number of two-dimensional equilibrium equations of the shell. When the numerical solution of a shell problem is needed, however, only a finite set of equations can be considered and solved efficiently. Depending on the number of the two-dimensional equilibrium equations chosen, a large variety of dimensionally reduced shell models can be derived, and hierarchic sets of shell models can also be constructed. In this section an equilibrated stress space for thin shells is derived. It is assumed that the stress boundary conditions on the shell faces are a priori satisfied, just like in the classical shell models, which means that the procedure described in Section 3.3 can be applied. 
4.1. Equilibrated stress space for thin shells. In the case of thin shells, let the first two equilibrium equations of (3.10) be selected and let the other equations, corresponding to the higher powers of $\xi^{3} \equiv \zeta$, be neglected with the assumption that they are identically satisfied. The two equations for $n=0$ and $n=1$ are

$$
\begin{aligned}
& \frac{1}{\sqrt{{ }_{0} A}}\left(\sqrt{{ }_{0} A}{ }_{0} \hat{\boldsymbol{p}}^{\lambda}\right)_{, \lambda}+{ }_{1} \hat{\boldsymbol{p}}^{3}+{ }_{0} \hat{\boldsymbol{b}}=\mathbf{0}, \\
& \frac{1}{\sqrt{{ }_{0} A}}\left(\sqrt{{ }_{0} A}{ }_{1} \hat{\boldsymbol{p}}^{\lambda}\right)_{, \lambda}+2{ }_{2} \hat{\boldsymbol{p}}^{3}+{ }_{1} \hat{\boldsymbol{b}}=\mathbf{0} .
\end{aligned}
$$

According to these equations, the first Piola-Kirchhoff stress vectors $\hat{\boldsymbol{p}}^{\ell}$, defined in (3.8), are approximated across the thickness of the shell by linear and quadratic polynomials in $\zeta$ :

$$
\begin{aligned}
& \hat{\boldsymbol{p}}^{\lambda}\left(\xi^{\alpha}, \zeta\right)={ }_{0} \hat{\boldsymbol{p}}^{\lambda}\left(\xi^{\alpha}\right)+{ }_{1} \hat{\boldsymbol{p}}^{\lambda}\left(\xi^{\alpha}\right) \zeta, \\
& \hat{\boldsymbol{p}}^{3}\left(\xi^{\alpha}, \zeta\right)={ }_{0} \hat{\boldsymbol{p}}^{3}\left(\xi^{\alpha}\right)+{ }_{1} \hat{\boldsymbol{p}}^{3}\left(\xi^{\alpha}\right) \zeta+{ }_{2} \hat{\boldsymbol{p}}^{3}\left(\xi^{\alpha}\right) \zeta^{2} .
\end{aligned}
$$

Following now the method applied in Section 3.3 the coefficients ${ }_{1} \hat{\boldsymbol{p}}^{3}$ and ${ }_{2} \hat{\boldsymbol{p}}^{3}$ can be expressed from the stress boundary conditions $(3.21)-(3.22)$ prescribed on the shell faces: in view of (4.4), equations (3.27)- 3.28 simplify to the form

$$
\begin{aligned}
{ }_{1} \hat{\boldsymbol{p}}^{3} & ={ }_{1} \widetilde{\boldsymbol{p}}, \\
{ }_{2} \hat{\boldsymbol{p}}^{3} & =\frac{4}{d^{2}}{ }_{0} \widetilde{\boldsymbol{p}}-\frac{4}{d^{2}}{ }_{0} \hat{\boldsymbol{p}}^{3} .
\end{aligned}
$$

Substituting (4.5)-(4.6) into 4.1)-(4.2), the equilibrium equations for the first PiolaKirchhoff stress vectors ${ }_{0} \hat{\boldsymbol{p}}^{\ell}$ and ${ }_{1} \hat{\boldsymbol{p}}^{\lambda}$ take the form

$$
\begin{aligned}
& \frac{1}{\sqrt{{ }_{0} A}}\left({\sqrt{0}{ }_{0}}_{0} \hat{\boldsymbol{p}}^{\lambda}\right)_{, \lambda}+{ }_{1} \widetilde{\boldsymbol{p}}+{ }_{0} \hat{\boldsymbol{b}}=\mathbf{0} \\
& \frac{1}{\sqrt{{ }_{0} A}}\left(\sqrt{{ }_{0} A}{ }_{1} \hat{\boldsymbol{p}}^{\lambda}\right)_{, \lambda}-\frac{8}{d^{2}}{ }_{0} \hat{\boldsymbol{p}}^{3}+\frac{8}{d^{2}}{ }_{0} \tilde{\boldsymbol{p}}+{ }_{1} \hat{\boldsymbol{b}}=\mathbf{0} .
\end{aligned}
$$

These equations can be deduced from 3.29 - 3.30, as well, by neglecting the higherorder terms. The equilibrated stress vectors, satisfying (4.7)-4.8 are obtained from (3.32)-(3.35):

$$
\begin{aligned}
& { }_{0} \hat{\boldsymbol{p}}^{\lambda}={ }_{0} \epsilon^{\lambda \beta}{ }_{0} \boldsymbol{\psi}_{, \beta}-\frac{1}{2 \sqrt{{ }_{0} A}}{ }_{1} \boldsymbol{f}^{\lambda}, \\
& { }_{0} \hat{\boldsymbol{p}}^{3}={ }_{0} \epsilon^{3 \lambda}{ }_{0} \boldsymbol{\chi}_{, \lambda}, \\
& { }_{1} \hat{\boldsymbol{p}}^{\lambda}={ }_{0} \epsilon^{\lambda \beta}{ }_{1} \boldsymbol{\psi}_{, \beta}+\frac{8}{d^{2}}{ }_{0} \epsilon^{3 \lambda}{ }_{0} \boldsymbol{\chi}-\frac{1}{2 \sqrt{{ }_{0} A}}{ }_{0} \boldsymbol{f}^{\lambda},
\end{aligned}
$$

where ${ }_{0} \boldsymbol{f}^{\lambda}$ and ${ }_{1} \boldsymbol{f}^{\lambda}$ are defined by $3.36-3.37$. From $4.9-4.11$ it follows that the power series of the stress function vectors (3.11)-3.12 are restricted now to linear and constant functions, according to

$$
\begin{aligned}
\boldsymbol{\psi}\left(\xi^{\alpha}, \zeta\right) & ={ }_{0} \boldsymbol{\psi}\left(\xi^{\alpha}\right)+{ }_{1} \boldsymbol{\psi}\left(\xi^{\alpha}\right) \zeta \\
\chi\left(\xi^{\alpha}, \zeta\right) & ={ }_{0} \boldsymbol{\chi}\left(\xi^{\alpha}\right),
\end{aligned}
$$


i.e., only three first-order stress function vectors, ${ }_{0} \boldsymbol{\psi},{ }_{1} \boldsymbol{\psi}$ and ${ }_{0} \chi$, are required in this model for thin shells.

The equilibrated first Piola-Kirchhoff stress components can be obtained from equations 3.46$)-(3.51$ of Section 3.4 by taking into account the stress function space 4.12 and 4.13):

$$
\begin{aligned}
{ }_{0} \hat{P}^{k \lambda} & ={ }_{0} \epsilon^{\lambda \beta}{ }_{0} \psi_{1 \beta}{ }_{1 \beta}-\frac{1}{2 \sqrt{{ }_{0} A}}{ }_{1}\left(f^{k}\right)^{\lambda}, \\
{ }_{1} \hat{P}^{k \lambda} & ={ }_{0} \epsilon^{\lambda \beta}{ }_{1} \psi_{\mid \beta}^{k}+\frac{8}{d^{2}}{ }_{0} \epsilon^{3 \lambda}{ }_{0} \chi^{k}-\frac{1}{2 \sqrt{{ }_{0} A}}{ }_{0}\left(f^{k}\right)^{\lambda}, \\
{ }_{0} \hat{P}^{k 3} & ={ }_{0} \epsilon^{3 \lambda}{ }_{0} \chi_{{ }_{1 \lambda}}^{k}, \\
{ }_{1} \hat{P}^{k 3} & ={ }_{1} \tilde{p}^{k}, \\
{ }_{2} \hat{P}^{k 3} & =\frac{4}{d^{2}}{ }_{0} \tilde{p}^{k}-\frac{4}{d^{2}}{ }_{0} \hat{P}^{k 3},
\end{aligned}
$$

where ${ }_{i}\left(f^{k}\right)^{\lambda}, i=1,2$ are the components of the load vectors 3.36)-3.37) with respect to the base vectors ${ }_{0} \boldsymbol{A}_{k}$. According to 4.14-4.18, the components $\hat{P}^{k \ell}$ of the first Piola-Kirchhoff stress tensor are approximated by linear and quadratic polynomials across the thickness:

$$
\begin{aligned}
& \hat{P}^{k \lambda}\left(\xi^{\alpha}, \zeta\right)={ }_{0} \hat{P}^{k \lambda}\left(\xi^{\alpha}\right)+{ }_{1} \hat{P}^{k \lambda}\left(\xi^{\alpha}\right) \zeta, \\
& \hat{P}^{k 3}\left(\xi^{\alpha}, \zeta\right)={ }_{0} \hat{P}^{k 3}\left(\xi^{\alpha}\right)+{ }_{1} \hat{P}^{k 3}\left(\xi^{\alpha}\right) \zeta+{ }_{2} \hat{P}^{k 3}\left(\xi^{\alpha}\right) \zeta^{2} .
\end{aligned}
$$

This approximation is consistent with the stress state obtained from the classical shell models and equivalent with the stresses obtained from the three-dimensional equilibrium equations through a posteriori integration across the thickness of the shell.

The shifted components $\bar{P}^{k \ell}$ of the first Piola-Kirchhoff stress tensor can be derived using relation (3.54). When the approximations 2.22 for the shifter tensor and its determinant are applied, the equality $\bar{P}^{k \ell}=\hat{P}^{k \ell}$ is valid for the shifted stress components.

4.2. Relationship with resultant-based shell equations. In this section, firstorder stress function vectors generating equilibrated stress resultants and stress couples used in classical shell theories are introduced and their relationships to the stress function vectors 4.12)-4.13 that generate equilibrated stress vectors are investigated.

4.2.1. Equilibrated stress resultants and stress couples. The vectorial equilibrium equations for the first Piola-Kirchhoff stress resultants and stress couples can be derived from the three-dimensional equilibrium equations (3.7), rewritten in the form

$$
\frac{1}{\sqrt{{ }_{0} A}}\left(\sqrt{{ }_{0} A} \mu \boldsymbol{p}^{\lambda}\right)_{, \lambda}+\left(\mu \boldsymbol{p}^{3}\right)_{3}+{ }^{0} \rho \mu \boldsymbol{b}=\mathbf{0} .
$$


Introducing the infinite number of first Piola-Kirchhoff stress resultant vectors as

$$
{ }_{i} \boldsymbol{N}\left(\xi^{\alpha}\right)=\int_{(d)} \zeta^{i} \mu \boldsymbol{p}^{\ell} \mathrm{d} \zeta, \quad i=0,1,2,3, \ldots,
$$

the 'classical' stress resultants and stress couples are obtained for $i=0$ and $i=1$ :

$$
\begin{gathered}
\boldsymbol{N}^{\ell}\left(\xi^{\alpha}\right):={ }_{0} \boldsymbol{N}^{\ell}=\int_{(d)} \mu \boldsymbol{p}^{\ell} \mathrm{d} \zeta, \\
\boldsymbol{M}^{\lambda}\left(\xi^{\alpha}\right):={ }_{1} \boldsymbol{N}^{\lambda}=\int_{(d)} \zeta \mu \boldsymbol{p}^{\lambda} \mathrm{d} \zeta .
\end{gathered}
$$

Note that ${ }_{1} \boldsymbol{N}^{3}=\boldsymbol{M}^{3}=0$.

Integrating equation 4.21 with respect to the thickness coordinate $\zeta$ and taking into account the stress boundary conditions on the faces, the vectorial equilibrium equation

$$
\frac{1}{\sqrt{{ }_{0} A}}\left(\sqrt{{ }_{0} A} N^{\lambda}\right)_{\lambda}+\mathfrak{p}=\mathbf{0}
$$

is obtained for the first Piola-Kirchhoff stress resultants $\boldsymbol{N}^{\lambda}$, where the load vector $\mathfrak{p}$ is given by

$$
\mathfrak{p}\left(\xi^{\alpha}\right)=\mu^{+0} \widetilde{\boldsymbol{p}}^{+}+\mu^{-0} \widetilde{\boldsymbol{p}}^{-}+\int_{(d)}{ }^{0} \rho \mu \boldsymbol{b} \mathrm{d} \zeta .
$$

Multiplying equation 4.21 by $\zeta$, integrating it then with respect to $\zeta$ and taking into account the stress boundary conditions on the faces again, the vectorial moment equilibrium equation is obtained:

$$
\frac{1}{\sqrt{{ }_{0} A}}\left(\sqrt{{ }_{0} A} M^{\lambda}\right)_{\lambda}-N^{3}+\mathfrak{m}=\mathbf{0}
$$

where the moment load vector $\mathfrak{m}$ is given by

$$
\mathfrak{m}\left(\xi^{\alpha}\right)=\frac{d}{2}\left(\mu^{+0} \widetilde{\boldsymbol{p}}^{+}-\mu^{-0} \widetilde{\boldsymbol{p}}^{-}\right)+\int_{(d)} \zeta^{0} \rho \mu \boldsymbol{b} \mathrm{d} \zeta .
$$

Equilibrium equations 4.25 and 4.27 can identically be satisfied by introducing three arbitrary but differentiable first-order stress function vectors, denoted by $\mathcal{F}\left(\xi^{\alpha}\right)$, $\mathcal{G}\left(\xi^{\alpha}\right)$ and $\mathcal{H}\left(\xi^{\alpha}\right)$, according to

$$
\begin{aligned}
\boldsymbol{N}^{\lambda} & ={ }_{0} \epsilon^{\lambda \beta} \mathcal{F},{ }_{\beta}-\frac{1}{2 \sqrt{{ }_{0} A}} \int_{\left(\xi^{\lambda}\right)} \sqrt{{ }_{0} A} \mathfrak{p} \mathrm{d} \xi^{\lambda}, \\
\boldsymbol{N}^{3} & ={ }_{0} \epsilon^{3 \lambda} \mathcal{G},{ }_{\lambda}, \\
\boldsymbol{M}^{\lambda} & ={ }_{0} \epsilon^{\lambda \beta} \mathcal{H}_{\beta}+{ }_{0} \epsilon^{3 \lambda} \mathcal{G}-\frac{1}{2 \sqrt{{ }_{0} A}} \int_{\left(\xi^{\lambda}\right)} \sqrt{{ }_{0} A} \mathfrak{m} \mathrm{d} \xi^{\lambda} .
\end{aligned}
$$

Note that in the works [15] and [16, the stress function vector $\mathcal{F}$ is already used to satisfy the membrane equilibrium equation 4.25, according to 4.29). However, the stress function vectors $\mathcal{G}$ and $\mathcal{H}$ are not introduced and used, for satisfying the bending equilibrium equation 4.27) in the works mentioned, as the bending moments were derived from the bending strains through constitutive equations. 
4.2.2. Relationships for the stress functions. The relationships between the first-order stress function vectors ${ }_{0} \psi\left(\xi^{\alpha}\right),{ }_{0} \chi\left(\xi^{\alpha}\right),{ }_{1} \boldsymbol{\psi}\left(\xi^{\alpha}\right)$, introduced in Section 4.1. and $\mathcal{F}\left(\xi^{\alpha}\right)$, $\mathcal{G}\left(\xi^{\alpha}\right), \mathcal{H}\left(\xi^{\alpha}\right)$ introduced above can be obtained as follows. Substituting (4.3)-4.4 into 4.23)-4.24, the relations between the stress vectors and the stress resultants are obtained:

$$
\begin{array}{rlrl}
{ }_{0} \hat{\boldsymbol{p}}^{\lambda} & =\frac{\boldsymbol{N}^{\lambda}}{d}, & { }_{1} \hat{\boldsymbol{p}}^{\lambda}=12 \frac{\boldsymbol{M}^{\lambda}}{d^{3}}, & \\
{ }_{0} \hat{\boldsymbol{p}}^{3}=\frac{3}{2} \frac{\boldsymbol{N}^{3}}{d}-\frac{1}{2}{ }_{0} \widetilde{\boldsymbol{p}}, & { }_{1} \hat{\boldsymbol{p}}^{3}={ }_{1} \widetilde{\boldsymbol{p}}, & { }_{2} \hat{\boldsymbol{p}}^{3}=\frac{6}{d^{2}}\left({ }_{0} \widetilde{\boldsymbol{p}}-\frac{\boldsymbol{N}^{3}}{d}\right) .
\end{array}
$$

Inserting 4.32 in 4.3 and 4.33 in 4.4 , the relations

$$
\begin{aligned}
\hat{\boldsymbol{p}}^{\lambda} & =\frac{\boldsymbol{N}^{\lambda}}{d}+12 \frac{\boldsymbol{M}^{\lambda}}{d^{3}} \zeta, \\
\hat{\boldsymbol{p}}^{3} & =\frac{3}{2} \frac{\boldsymbol{N}^{3}}{d}\left(1-\frac{4}{d^{2}} \zeta^{2}\right)-{ }_{0} \widetilde{\boldsymbol{p}}\left(\frac{1}{2}-\frac{6}{d^{2}} \zeta^{2}\right)+{ }_{1} \widetilde{\boldsymbol{p}} \zeta
\end{aligned}
$$

are obtained, which are partially known from the classical shell theories.

The relationships between the first-order stress function vectors $\mathcal{F}, \mathcal{G}, \mathcal{H}$ and ${ }_{0} \boldsymbol{\psi}$, ${ }_{0} \boldsymbol{\chi},{ }_{1} \boldsymbol{\psi}$ are obtained by substituting $4.29-4.31$ into $4.32-4.33$ and comparing the result with 4.5- 4.6 and 4.9-(4.11):

$$
\begin{gathered}
{ }_{0} \boldsymbol{\psi}=\frac{\mathcal{F}}{d}, \\
\boldsymbol{\psi}=12 \frac{\mathcal{H}}{d^{3}} \\
{ }_{0} \epsilon^{3 \lambda}\left({ }_{0} \boldsymbol{\chi}-\frac{3}{2} \frac{\mathcal{G}}{d}\right){ }_{, \lambda}+\frac{1}{2}{ }_{0} \widetilde{p}=\mathbf{0} .
\end{gathered}
$$

On inserting the relations 4.36 and 4.37 in 4.12, it follows that

$$
\psi\left(\xi^{\alpha}, \zeta\right)=\frac{\mathcal{F}}{d}+12 \frac{\mathcal{H}}{d^{3}} \zeta
$$

and, thus,

$$
\begin{aligned}
\mathcal{F}\left(\xi^{\alpha}\right) & =\int_{(d)} \psi\left(\xi^{\alpha}, \zeta\right) \mathrm{d} \zeta \\
\mathcal{H}\left(\xi^{\alpha}\right) & =\int_{(d)} \zeta \boldsymbol{\psi}\left(\xi^{\alpha}, \zeta\right) \mathrm{d} \zeta
\end{aligned}
$$

which indicate that $\mathcal{F}$ and $\mathcal{H}$ can be considered as two-dimensional stress function resultants obtained from the three-dimensional stress function vector $\boldsymbol{\psi}$.

Equation 4.38 is a partial differential equation for ${ }_{0} \mathcal{\chi}$ and $\mathcal{G}$. For the special case when the loads on the shell faces are zero, the relation

$$
{ }_{0} \chi=\frac{3}{2} \frac{\mathcal{G}}{d}
$$

holds for them. 


\section{Concluding Remarks}

The equilibrated stress space given in this paper for nonlinear deformation of shells can serve as a starting point for the derivation of a stress-based nonlinear shell theory and a dual-mixed, complementary energy-based finite element model for shells. The variational background of this theory and the related finite element model is Fraeijs de Veubeke's variational principle for 3D elasticity problems [31. The dimensional reduction procedure for the present shell model relies on a priori assumptions regarding the variation of the stress space across the thickness, instead of the standard Kirchhoff-Love or Reissner-Mindlin type kinematical assumptions on the deformed geometry of the shell. Since the transverse shear and the transverse normal stresses are also present in the formulation, three-dimensional constitutive equations can directly be applied. Beside this fact, the main advantage of the stress-based models and the related dual-mixed finite elements for shells is their expected locking-free behavior, i.e., their superior performance in those cases, when the classical, displacement-based models and elements exhibit different types of numerical over-stiffening phenomenon.

\section{REFERENCES}

1. B. M. Fraeijs de Veubeke. "Diffusive equilibrium models." Lecture notes for the International Research Seminar on 'The theory and application of finite element methods', University of Calgary, Alberta, Canada (1973).

2. B. M. Fraeijs de Veubeke. "Stress function approach." Proceedings of the World Congress on Finite Element Methods. Bournemouth, U.K., 1975, J.1-J.51.

3. B. M. Fraeijs de Veubeke. "Discretization of rotational equilibrium in the finite element method." Lecture Notes in Mathematics. Proceedings of the Mathematical Aspects of Finite Element Methods held in Rome, December 10-12, 1975. Ed. by I. Galligiani and E. Magenes. Vol. 606. Berlin: Springer-Verlag, 1977, pp. 87-112. DOI: 10.1007/BFb0064451.

4. I. Kozák and G. Szeidl. "The field equations and the boundary conditions with force stresses and couple stresses in the linearized theory of micropolar elastostatics." Acta Technica Academiae Scientiarum Hungaricae, 91(1-2), (1980), pp. $57-80$.

5. E. Bertóti. "Indeterminacy of first-order stress functions and the stress- and rotation-based formulation of linear elasticity." Computational Mechanics, 14, (1994), pp. 249-265. DOI: 10.1007/BF00370076.

6. L. E. Malvern. Introduction to the Mechanics of a Continuous Medium. Englewood Cliffs, New Jersey: Prentice-Hall, 1969.

7. B. M. Fraeijs de Veubeke and A. Millard. "Discretization of stress fields in the finite element method." Journal of the Franklin Institute, 302, (1976), pp. 389412. DOI: $10.1016 / 0016-0032$ (76)90032-6.

8. H. Murakawa and S. N. Atluri. "Finite elasticity solutions using hybrid finite elements based on a complementary energy principle." Journal of Applied Mechanics, 45(3), (1978), pp. 539-547. DOI: 10.1115/1.3424358. 
9. H. Murakawa and S. N. Atluri. "Finite elasticity solutions using hybrid finite elements based on a complementary energy principle. Part 2: Incompressible materials." Journal of Applied Mechanics, 46(1), (1979), pp. 71-77. DOI: 10 . $1115 / 1.3424531$.

10. E. Bertóti. "On the stress function approach in three-dimensional elasticity." Acta Mechanica, 190(1-4), (2007), pp. 197-204. DOI: 10.1007/s00707-0060432-6.

11. H. Schaefer. "Die Analogie zwischen den Verschiebungen und den Spannungsfunktionen in der Biegetheorie der Kreiszylinderschale." Ingenieur-Archiv, 29, (1960), pp. 125-133.

12. W. Günther. "Analoge Systeme von Schalengleichungen." Ingenieur-Archiv, 30, (1961), pp. 160-186. DOI: 10.1007/BF00534754.

13. A. E. Goldenveizer. Theory of Elastic Thin Shells. Oxford: Pergamon Press, 1961.

14. Y. Yamamoto. "An intrinsic theory of shells." International Journal of Solids and Structures, 2, (1966), pp. 235-248. DOI: 10.1016/0020-7683(66)90017-5.

15. J. G. Simmonds and D. A. Danielson. "Nonlinear shell theory with finite rotation and stress function vectors." Journal of Applied Mechanics, 39(4), (1972), pp. 1085-1090. DOI: 10.1115/1.3422833.

16. S. N. Atluri. "Alternate stress and conjugate strain measures, and mixed variational formulations involving rigid rotations, for computational analyses of finitely deformed solids, with application to plates and shells - I. Theory." Computers and Structures, 18, (1984), pp. 93-116. DOI: 10.1016/0045-7949(84) 90085-3.

17. E. F. Punch and S. N. Atluri. "Large displacement analysis of plates by a stress-based finite element approach." Computers and Structures, 24(1), (1986), pp. 107-117. DOI: 10.1016/0045-7949(86)90339-1.

18. R. Valid. The Nonlinear Theory of Shells through Variational Principles: From Elementary Algebra to Differential Geometry. Chichester: John Wiley \& Sons, 1995.

19. L. G. Kocsán. "Derivation of a dual-mixed $h p$-finite element model for axisymmetrically loaded cylindrical shells." Archive of Applied Mechanics, 81(12), (2011), pp. 1953-1971. DOI: 10.1007/s00419-011-0530-3.

20. B. Tóth and L. G. Kocsán. "Comparison of dual-mixed $h$ - and $p$-version finite element models for axisymmetric problems of cylindrical shells." Finite Element in Analysis and Design, 65, (2013), pp. 50-62. DOI: 10.1016/j.finel.2012. 11.002 .

21. E. Reissner. "The effect of transverse shear deformations on the bending of elastic plates." Journal of Applied Mechanics, 12, (1945), A69-A77.

22. R. D. Mindlin. "Influence of rotatory inertia and shear on flexural motions of isotropic elastic plates." Journal of Applied Mechanics, 18, (1951), pp. 31-38.

23. P. M. Naghdi. Foundations of Elastic Shell Theory. In Progress in Solid Mechanics, Vol. IV (I. N. Sneddon and R. Hill, Eds.) pp. 1-90. Amsterdam: NorthHolland, 1963. 
24. P. M. Naghdi. The Theory of Shells and Plates. In Handbuch der Physik, Band VIa/2 (S. Flügge and C. Truesdell, Eds.) Berlin: Springer-Verlag, 1972, pp. 425640. DOI: $10.1007 / 978-3-662-39776-3 \_5$.

25. G. Kirchhoff. "Über das Gleichgewicht und die Bewegung einer elastischen Scheibe." Journal für die reine und angewandte Mathematik, 40, (1850), pp. 51-58.

26. A. E. H. Love. "The small free vibrations and deformations of a thin elastic shell." Philosophical Transactions of the Royal Society of London Series A, 179, (1888), pp. 491-546.

27. V. V. Novozhilov. Thin Shell Theory. (2nd ed., Wolters-Noordhoff, Groningen, 1970). Groningen: Noordhoff, 1959.

28. J. L. Sanders. "Non-linear theories for thin shells." Quarterly of Applied Mathematics, 21, (1963), pp. 21-36.

29. W. T. Koiter. "On the foundations of the linear theory of thin elastic shells. III." Proceedings of the Koninklijke Nederlandse Akademie van Wetenschappen, B73, (1970), pp. 169-195.

30. C. Truesdell and R. A. Toupin. The Classical Field Theories. In Handbuch der Physik, Band III/1 (S. Flügge, Ed.) Berlin: Springer-Verlag, 1960. DOI: 10.1007/ 978-3-642-45943-6_2.

31. B. M. Fraeijs de Veubeke. "A new variational principle for finite elastic displacements." International Journal for Engineering Sciences, 10, (1972), pp. 745763. DOI: $10.1016 / 0020-7225$ (72)90079-1. 